\title{
Recurrent Medullary Carcinoma detected by Gallium-68 Positron Emission Tomography
}

\author{
Cigdem Soydal, Elgin Ozkan, Mine Araz, Ozlem N Kucuk, Taner Demirer
}

\begin{abstract}
In this case, we would like to share our experience of a recurrent medullary thyroid cancer patient whose recurrence was detected by Ga-68 DOTATATE PET/CT.
\end{abstract}

Keywords: Medullary thyroid cancer, Recurrent disease, Ga-68 DOTATATE PET/CT.

How to cite this article: Soydal C, Ozkan E, Araz M, Kucuk ON, Demirer T. Recurrent Medullary Carcinoma detected by Gallium-68 Positron Emission Tomography. World J Endoc Surg 2013;5(2):59-60.

\section{Source of support: Nil}

Conflict of interest: None

\section{INTRODUCTION}

After primary surgery, detection of medullary thyroid cancer (MTC) recurrence can be problematic in patients with elevated calcitonin levels. It recommended that patients with calcitonin levels higher than $150 \mathrm{pg} / \mathrm{ml}$ should be scanned by imaging modalities. ${ }^{1}$ Neck ultrasound (US) is the first scanning tool in this case. However, it can be difficult to evaluate neck by US, especially in patients who underwent repeated surgery. Most nuclear medicine techniques, such as Tl-201 chloride, Tc-99m sestamibi, Tc-99m (V)-DMSA, I-123/I-131 MIBG, In-111 pentetreotide and 18F-FDG PET/CT have been researched in MTC recurrence. ${ }^{2-5}$ Although PET/CT has a higher spatial resolution than SPECT imaging, sensitivity of 18F-FDG PET/CT is relatively low in MTC due to well differentiation rate of MTCs. Imaging tools with Ga-68 labeled somatostatin analogs have widely been performed in neuroendocrine tumors and also there is limited literature about the usage in medullary thyroid carcinoma.,

\section{MATERIALS AND METHODS}

We present a case of 35 years old female patient who underwent Ga-68 DOTATATE PET/CT for restaging of medullary thyroid carcinoma. Before 14 years she had undergone subtotal thyroidectomy for multinodular goiter. Pathological examination of this thyroidectomy material was reported as benign. After 5 years, a lymph node excision was performed and the patient has been diagnosed as medullary thyroid cancer. After completion of thyroidectomy, the patient has been operated twice for neck lymph node metastases until today. The patient was referred for Ga-68 DOTATATE PET/CT, for restaging of disease because of elevated serum calcitonin levels $(7400 \mathrm{pg} / \mathrm{ml})$. Before Ga-68 DOTATATE PET/CT, neck USG, thoracoabdominal CT, I-123 MIBG scintigraphy and 18FFDG PET/CT were performed and all of them were negative for recurrence. Whole body MIP image and transaxial CT and 18F-FDG PET images of thorax were shown in Figures 1 and 2. In Ga-68, DOTATATE intense Ga-68 DOTATATE accumulation which assumed to disease recurrence was seen in paratracheal, precarinal, prevascular and right hilar lymph nodes. Ga-68 DOTATATE PET/CT whole body MIP image and transaxial CT and PET images of thorax were shown in Figures 3 and 4.

\section{CONCLUSION}

In this case, we would like to contribute this limited literature by introducing intense Ga-68 DOTATATE uptake in recurrent MTC patient.

\section{REFERENCES}

1. Kloos RT, Eng C, Evans DB, et al. Medullary thyroid cancer: management guidelines of the American Thyroid Association. American Thyroid Association Guidelines Task Force, Thyroid. 2009 Jun;19(6):565-612.

2. Clarke S. Medullary thyroid cancer. Nuclear medicine in clinical diagnosis and treatment. 3rd ed. London: Churchill Livingstone; 2004;P165-174.

3. Hoegerle S, Altehoefer C, Ghanem N, et al. 18F-DOPA positron emission tomography for tumour detection in patients with medullary thyroid carcinoma and elevated calcitonin levels. Eur J Nucl Med 2001 Jan;28(1):64-71.

4. Brandt-Mainz K, Müller SP, Görges R, et al. The value of fluorine-18 fluorodeoxyglucose PET in patients with medullary thyroid cancer. Eur J Nucl Med 2000 May;27(5): 490-496.

5. de Groot JW, Links TP, Jager PL, et al. Impact of 18F-fluoro-2deoxy-D-glucose positron emission tomography (FDG-PET) in patients with biochemical evidence of recurrent or residual medullary thyroid cancer. Ann Surg Oncol 2004 Aug;11(8): 786-794.

6. Pałyga I, Kowalska A, Gǵsior-Perczak D, et al. The role of PETCT scan with somatostatin analogue labelled with gallium-68 $\left({ }^{68} \mathrm{Ga}\right.$-DOTATATE PETCT) in diagnosing patients with disseminated medullary thyroid carcinoma (MTC). Endokrynol Pol 2010 Sep-Oct;61(5):507-511. 


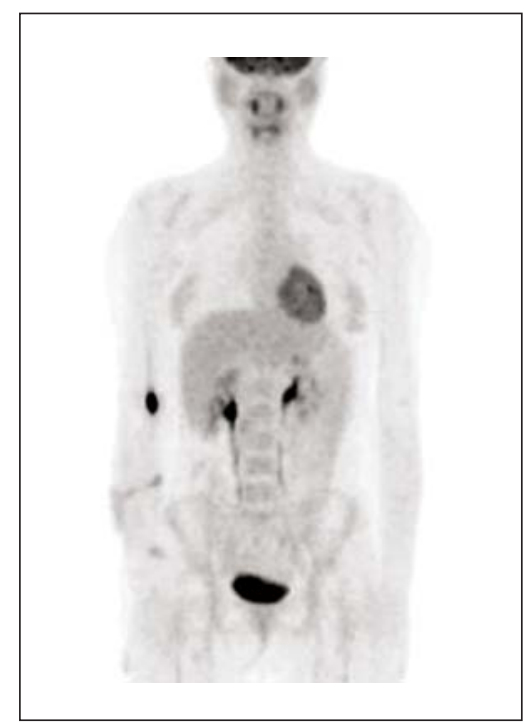

Fig. 1: 18F-FDG PET/CT whole body MIP image of the patient
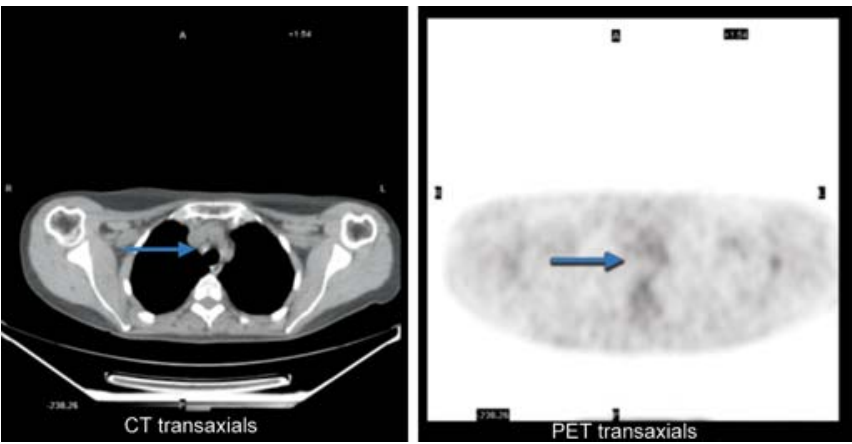

Fig. 2: Transaxial thoracic 18F-FDG CT and PET images of the patient

7. Traub-Weidinger T, Von Guggenberg E, Dobrozemsky G, et al. Preliminary experience with (68)Ga-DOTA-lanreotide positron emission tomography. QJ Nucl Med Mol Imaging 2010 Feb; 54(1):52-60.

\section{ABOUT THE AUTHORS}

\section{Cigdem Soydal (Corresponding Author)}

Consultant, Department of Nuclear Medicine, Ankara University Medical School, Mamak, Turkey, e-mail: csoydal@yahoo.com

\section{Elgin Ozkan}

Consultant, Department of Nuclear Medicine, Ankara University Medical School, Mamak, Turkey

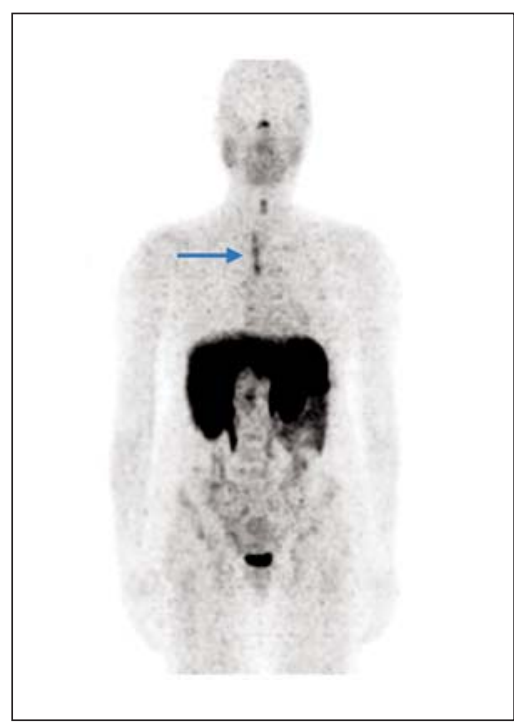

Fig. 3: Ga-68 DOTATATE PET/CT whole body MIP image of the patient

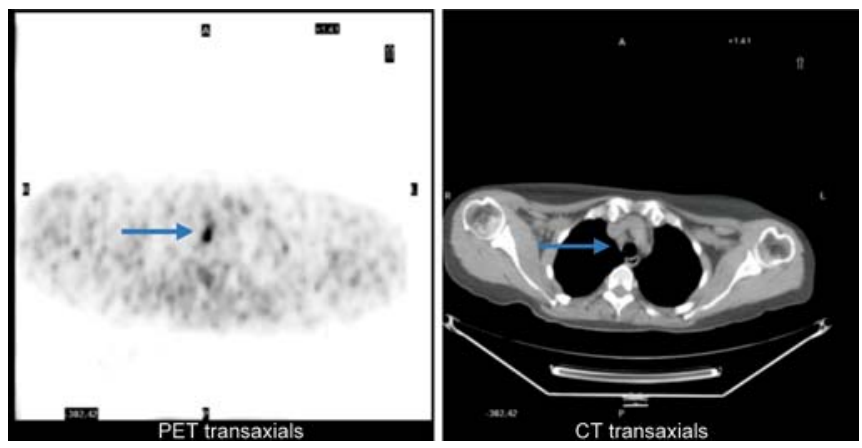

Fig. 4: Transaxial thoracic 18F-FDG CT and PET images of the patient

\section{Mine Araz}

Resident, Department of Nuclear Medicine, Ankara University Medical School, Mamak, Turkey

\section{Ozlem N Kucuk}

Professor, Department of Nuclear Medicine, Ankara University Medical School, Mamak, Turkey

\section{Taner Demirer}

Professor, Department of Hematology, Ankara University Medical School, Mamak, Turkey 